\title{
La reconversion des friches militaires urbaines dans les années 2000 : une opportunité pour Mulhouse?
}

The reconversion of the military urban brownfields in the 2000s: an opportunity for Mulhouse?

Die Requalifizierung der militärischen Ödländer in den 2000er Jahren: eine Chance für Mulhouse?

\section{Stéphanie Lotz-Coll}

\section{OpenEdition}

Journals

Édition électronique

URL : http://journals.openedition.org/rge/7330

DOI : 10.4000/rge.7330

ISSN : $2108-6478$

Éditeur

Association des géographes de l'Est

\section{Référence électronique}

Stéphanie Lotz-Coll, «La reconversion des friches militaires urbaines dans les années 2000 : une opportunité pour Mulhouse? », Revue Géographique de l'Est [En ligne], vol. 57 / 3-4 | 2017, mis en ligne le 29 octobre 2018, consulté le 10 décembre 2020. URL : http://journals.openedition.org/rge/7330 ; DOI : https://doi.org/10.4000/rge.7330

Ce document a été généré automatiquement le 10 décembre 2020.

Tous droits réservés 


\section{La reconversion des friches militaires urbaines dans les années 2000 : une opportunité pour Mulhouse?}

The reconversion of the military urban brownfields in the 2000s: an opportunity for Mulhouse?

Die Requalifizierung der militärischen Ödländer in den 2000er Jahren: eine

Chance für Mulhouse?

Stéphanie Lotz-Coll

1 Le phénomène de friches est lié à la ville, les évolutions de la société rendant progressivement obsolètes certains édifices. De facto, la génération de friches urbaines peut s'inscrire dans deux grands types de mutations urbaines (Piron, 2002), celles progressives, résultant de choix individuels, et les mutations brutales pour lesquelles la fonction du secteur urbain où était située la friche perd brusquement sa cohérence. Ceci est particulièrement vérifié pour les friches militaires urbaines, espaces fermés et réglementés qu'il faut intégrer à la ville à la cessation de leur activité initiale. Des contraintes socio-économiques s'ajoutent également à cet aspect urbain :

- Le site militaire n'avait qu'une activité très réduite avec un nombre limité de personnels depuis longtemps. Les effets induits de sa fermeture seront limités et il n'est pas nécessaire de rechercher rapidement des solutions économiques pour pallier le départ des derniers militaires ;

- Le site militaire employait beaucoup de personnels: sa cessation d'activité a des conséquences socio-économiques que la commune va s'efforcer de compenser au plus vite dans le cadre des dispositifs favorables mis en place par l'Etat pour les friches les plus récentes.

Dans les deux cas, la cession, surtout pour un site militaire avec une superficie importante, contraint les acteurs locaux à repenser la ville car le site militaire avait ses 
propres contraintes et formait une ville dans la ville. Il va s'agir d'envisager une remise en cohérence urbaine et la municipalité doit se positionner rapidement pour un éventuel achat pour ne pas laisser le site tomber en déshérence et au final de le réintégrer pleinement dans le tissu urbain. Au-delà de cet aspect pratique, la mise à jour de l'ensemble des documents d'urbanisme réglementaires est souvent nécessaire. La question se pose également pour des sites militaires de moindre ampleur car la ville a une priorité d'achat dans le cadre de leur cession par le ministère des Armées ${ }^{1}$.

Quelle que soit la période de cession du site, ce nouveau foncier disponible, rarement anticipé fait donc l'effet d'une "surprise de planification urbaine» (Lotz, 2017). En effet, alors que les années 1990 ont vu le développement de la notion de projet urbain et que les années 2000 ont davantage systématisé la mise en place d'un urbanisme réglementaire, le changement de fonction et l'éventuel besoin socio-économique d'une reconversion rapide du site peuvent bouleverser la planification urbaine à court ou moyen termes (Cohen-Grillet, 2008,). Les notions de réversibilité des projets déjà initiés et de complémentarité avec les opérations en cours peuvent alors devenir déterminantes. Cette surprise de planification urbaine ici appliquée aux friches militaires pourrait concerner toute cessation d'activités relativement rapide.

La Défense, parfois présentée comme aménageur du territoire (Regrain, 1988) a, de fait, contraint les aménagements du territoire civil pour des raisons géostratégiques (Grellety-Bosviel, 1970). Pour une ville, le départ de l'armée d'une ville, même s'il rompt une logique urbaine, peut donc être ressenti avec pessimisme, mais il peut aussi se présenter comme une opportunité. Ainsi, la reconversion des friches militaires de Freiburg im Breisgau (Allemagne), Montpellier ou Grenoble a eu un impact positif en termes d'image, de développement économique et social et de dynamisme démographique. Ces requalifications ont d'ailleurs eu un effet levier pour l'ensemble du territoire (Janin et Andres, 2008).

5 Cet article propose, dans un premier temps, un focus sur la friche comme objet urbain avant de s'interroger sur la possible banalisation d'une friche militaire lors de sa cession. Il s'agira alors de se référer aux possibilités de reconversion que ces sites peuvent offrir sur un plan foncier dans le cadre d'une approche qui n'est pas géohistorique et ne comprend qu'a minima une vision patrimoniale. Dans un dernier temps, l'exemple de la reconversion des casernes de Mulhouse permettra d'envisager dans quelle mesure les projets de reconversion des friches militaires peuvent être des leviers d'attractivité s'ils répondent aux besoins du territoire et à l'enjeu urbanistique de renouvellement urbain.

\section{La friche, un objet urbain avec ses caractéristiques}

6 La friche et sa reconversion recouvrent des problématiques diverses. Les observations réalisées sur différents sites permettent de confirmer la régularité d'apparition de trois phases (Ambrosino et Andres, 2008) et d'envisager un quatrième temps peu mis en valeur jusqu'à présent (doc. 1) : 
Document $\mathrm{n}^{\circ} 1:$ les temps de la friche.

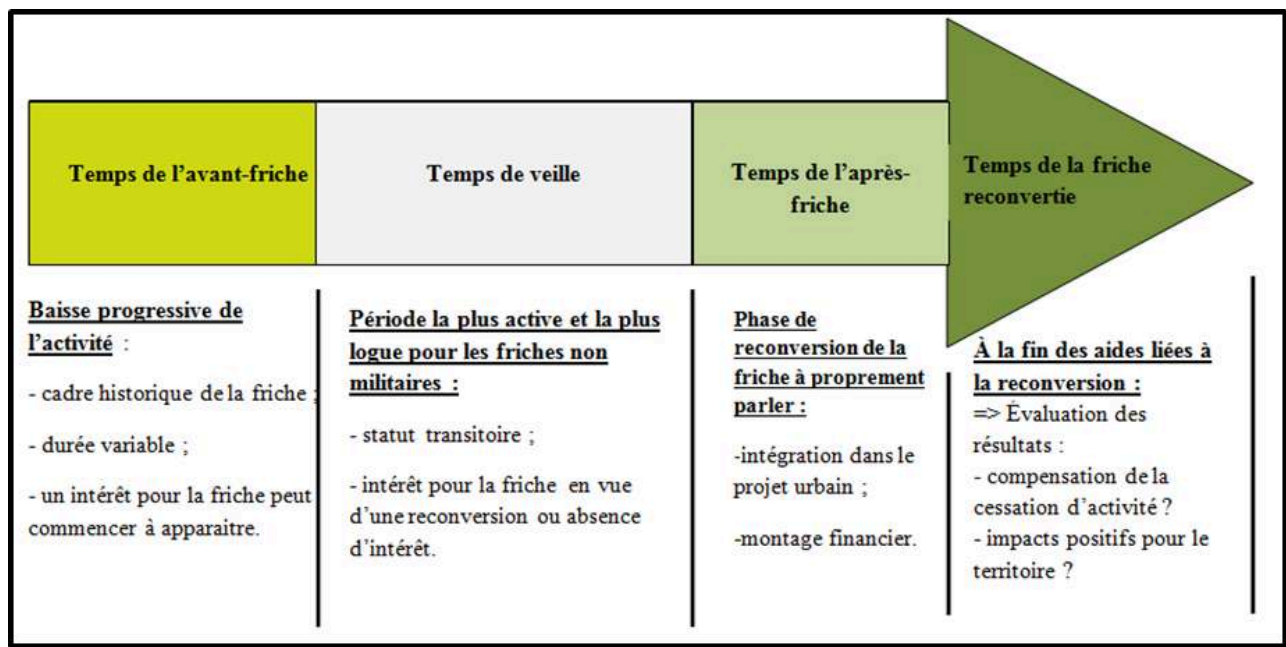

Réalisation par l'auteur

- L'avant-friche, une période transitoire caractérisée par une baisse progressive de l'activité du site sans changement dans sa fonction initiale, constitue le cadre historique de la friche. En fonction de sa durée, une situation de crise et de déclin généralisée peut avoir un impact sur l'ensemble de l'espace urbain associé à cette friche. Dans le cas des friches militaires, les cessions antérieures à 2008 ont parfois été longues (une dizaine d'années). Cette officialisation tardive de la décision de vente du site à l'issue de sa mise en sommeil a pu compliquer l'appréhension de cette période d'avant-friche (Guelton, 2001). À l'inverse, les cessions plus récentes sont souvent très rapides avec une échéance de un à quatre ans. Cette brutalité laisse alors peu d'opportunités pour des questionnements sur la pertinence de la fonctionnalité économique, technique ou spatiale de la friche.

7 Quelle que soit son origine, la friche prend une importance accrue, pour deux acteurs centraux, la collectivité locale et le propriétaire qui définissent alors, conjointement ou non, des orientations initiales pour la friche et son éventuel temps de veille. L'éventualité de la rentabilité du site à la vente est abordée, la friche peut, dès lors devenir une ressource foncière et immobilière.

- La veille est la période la plus active et normalement la plus longue. Durant cette phase transitoire entre l'abandon du site et son projet de réutilisation (planifié), les friches bénéficient en général d'un statut provisoire « où malgré le jeu des délimitations foncières, la vocation de l'espace, ni privé ni public, s'y trouve brouillée » (Robins des villes, 2008). La friche est à la fois symbole de permissivité pour des milieux marginaux et de ressource foncière pour la ville; le développement d'activités temporaires peut alors remettre en question le déroulement ultérieur de la reconversion du site comme ce fut le cas avec la démarche conceptuelle die "Selbstorganisierte unabhängige Siedlungsinitiative » (SUSI) pour le quartier Vauban à Freiburg im Breisgau (Allemagne) ${ }^{2}$.

8 C'est un temps de réflexion sur les possibilités d'évolution de la friche. En France, pour les friches militaires récentes, c'est le moment où le site va devenir accessible pour envisager son potentiel et déterminer les contraintes de remise en état qu'il sera nécessaire de négocier avec la Défense. Cet aspect sera développé ultérieurement.

9 L'après friche est, à proprement parler, la phase de reconversion du site, les différents acteurs réajustent alors les paramètres issus de la phase de veille. Ces stratégies liées à l'attractivité du site à reconvertir sont enrichies et s'ouvrent à de nouveaux acteurs. Il 
arrive alors que les friches soient utilisées comme des éléments de marketing urbain pour répondre aux enjeux de concurrence entre les villes.

La friche devient alors une ressource foncière pour une ville durable respectueuse de l'environnement, socialement et économiquement inclusive, et qui se veut sobre quant à son impact sur les ressources et résiliente dans les chocs et les crises ${ }^{3}$.

11 Ces trois phases existent quel que soit le statut de la friche concernée. Cependant, leur durée et la nature des acteurs impliqués sont variables.

12 Dans le cadre des travaux sur l'effet levier qui désigne une technique destinée à multiplier les profits et les pertes sur la base d'un investissement initial, un quatrième temps de la friche a dû être envisagé lorsque les projets de l'après-friche ont abouti : il s'agit du temps de la friche reconvertie qui permet d'évaluer les effets de sa reconversion, en termes économique, social et urbain. (Lotz, 2017). En effet, compte tenu des investissements souvent réalisés, il importe que la réussite de la reconversion du site marque durablement le territoire, pour que cet espace ne redevienne pas une friche et pour faciliter la reconversion d'autres friches en appliquant un processus de planification déjà éprouvé. Dans le cadre de la reconversion des sites de défense, il pourra s'agir de l'effet potentiel qu'aura pu avoir la réalisation du projet urbain de reconversion de la friche militaire. En se basant sur les diagnostiques précédant le départ de l'armée, le nouvel espace doit alors répondre à de nombreuses questions :

- Les statistiques en termes de production d'emplois et de richesse montrent-elles que l'impact du départ de l'activité initiale a été compensé?

- Les données de l'INSEE en termes démographiques et de revenus locaux montrent-elles une revitalisation/redynamisation économique et sociale du territoire?

- L'emprise reconvertie s'intègre-t-elle dans le projet de la ville et a-t-elle acquis une nouvelle dimension urbaine de façon générale?

Ce dernier temps est souvent sous-estimé car les autorités considèrent parfois que l'opération est un succès lorsque l'espace a été requalifié. Ceci est d'autant plus vérifié lorsqu'il existe un décalage temporel entre le moyen terme du mandat électif et le long terme de la politique foncière et de la planification urbaine.

Dans le cas des friches militaires, les pouvoirs publics locaux sont fréquemment les premiers à s'intéresser au site car ils bénéficient d'une priorité pour l'acquérir. Au-delà du fait d'éviter d'avoir un site urbain dont la sécurisation serait difficile à assurer, il s'agit alors de déterminer l'intérêt de la friche militaire une fois reconvertie. La reconversion d'une friche militaire par la ville n'est pas acquise a priori mais elle doit s'envisager dans le cadre d'un projet d'ensemble pour la ville. Pour cela, un contexte global doit être défini, il s'agit du projet urbain.

\section{Les friches militaires, entre spécificité et banalisation}

15 En se multipliant, les friches ont eu un effet répulsif sur les collectivités : elles portaient les stigmates de la crise des systèmes de production et représentaient une rupture trop importante par rapport aux modèles de la société de consommation qui n'envisageaient alors que peu le recyclage (Lacaze, 2006). Pourtant, l'inversion de cette tendance incite certains propriétaires de friches à les valoriser comme réponses à la pénurie de foncier disponible. Depuis 6 ans, L'État français a ainsi mis en vente plus de 2000 biens dans le 
cadre de la Modernisation de l'Action Publique (MAP) afin de rationaliser son patrimoine tout en alimentant les caisses publiques et en réformant certaines d'administrations avec des regroupements de services. Au début des années 2010, les principales administrations concernées par ces ventes étaient les ministères de la défense (30\%), du budget (26\%) et de l'éducation $(22 \%)^{4}$.

Bien que ses friches fassent l'objet d'une catégorisation spécifique, l'offre immobilière du ministère des Armées n'a pas toujours été réalisée dans un contexte formalisé (Carré, 2001). Cependant, alors que les infrastructures telles que des tribunaux ou des écoles se banalisent assez facilement, l'offre de la Défense est hétéroclite et s'inscrit parfois dans des cadres spécifiques, tant en termes juridiques que financiers. Cela participe-t-il à faire de la friche militaire un cas particulier? Dans un contexte général qui conserve de la défiance vis-à-vis des friches du fait des risques potentiels liés à leur reconversion, une étude exhaustive des éventuelles spécificités de la friche militaire permet d'apporter une réponse complète.

Souvent méconnue du fait du secret entourant les activités de l'armée, la nature des friches militaires mérite d'être précisée car, en fonction des situations, elles peuvent avoir des éléments communs avec d'autres friches. Au milieu des années 1980, le ministère de la défense possédait un dixième du domaine public de l'État. Entre 2000 et 2006, plus de 600 sites ont été mis en vente pour un total de 6000 hectares. Ce mouvement s'accentue avec les Lois de Programmation Militaire (LPM) suivantes. Du fait de l'importance du patrimoine immobilier mis sur le marché et du besoin de rééquilibrage du budget de l'État, ces sites sont parfois moins facilement négociables que des biens courants. Cependant, ceci ne constitue pas une spécificité de la friche militaire, toutes les friches publiques étant soumises à cette logique de vendre au meilleur prix pour l'État.

En 2014, le ministère de la défense possédait encore le deuxième domaine foncier de France, avec 248000 hectares, soit 0,5\% du territoire (SGA, 2014).

\section{A. La friche militaire, une exception?}

19 Le ministère des Armées et les structures qui l'ont précédé ont toujours adapté leur patrimoine aux bouleversements géostratégiques, mais la Défense ne réorganise réellement ses implantations que depuis 1986 avec la vente de terrains dont elles n'avaient plus l'usage. C'est un paradoxe d'affirmer que les immeubles militaires témoignent d'une certaine longévité. C'est un héritage historique car, du XVIIIème siècle jusqu'au milieu du XXème siècle, la recrudescence des conflits armés avait eu pour conséquence l'implantation des multiples installations militaires au sein de zones urbaines ou sur ce qui était alors leur périphérie. Dès lors, ces sites ont pour la population une valeur historique, tant sur le plan architectural que mémorial, car ils font partie intégrante de la ville.

20 Au début des années 1980, environ 19000 hectares de l'ensemble des friches militaires étaient situés en agglomération ou en première couronne. Entre 1987 et 1997, 610 cessions de terrains militaires ont été réalisées pour un montant moyen de 250 millions de francs par $a^{5}$. La plupart des régions d'implantation militaire traditionnelle ont alors connu des fermetures de sites et des reconversions foncières. Ces dix années de vente de terrains militaires ont fait l'objet d'une mise en perspective qui a permis une ébauche de classification (Guelton, 1998). Cette dernière reste aujourd'hui valable dans 
le cadre des nouvelles LPM : trois grands ensembles se distinguent en fonction de la facilité à les reconvertir (doc. 2).

Document 2 : la friche militaire, une friche comme une autre selon sa catégorie.

\begin{tabular}{|c|c|c|}
\hline & $\begin{array}{l}\frac{1^{\text {er }} \text { ensemble: }}{\text { Des friches marquées }} \\
\text { par leur activité } \\
\text { initiale et non urbaines }\end{array}$ & $\begin{array}{l}\text { Friches trop marquées } \\
\text { par leur activité initiale } \\
\text { pour être assimilables à } \\
\text { une friche quelconque. }\end{array}$ \\
\hline $\begin{array}{c}\text { Ensemble } \\
\text { des } \\
\text { friches militaires }\end{array}$ & $\begin{array}{l}\frac{2^{\text {eme }} \text { ensemble : }}{\text { Des friches militaires }} \\
\text { bâties et urbaines }\end{array}$ & $\begin{array}{l}\text { Friches assimilables à } \\
\text { n'importe quelle friche } \\
\text { urbaine. }\end{array}$ \\
\hline Légende & & \\
\hline $\begin{array}{l}\text { Friches strictement } \\
\text { Militaires. } \\
\text { Friches urbaines } \\
\text { a banaliser. }\end{array}$ & $\begin{array}{c}\frac{3^{\text {eme }} \text { ensemble }}{\text { Des emprises urbaines }} \\
\text { partiellement bâties }\end{array}$ & $\begin{array}{l}\text { Des friches constituant } \\
\text { des opportunités } \\
\text { foncières communes à } \\
\text { toutes friches. }\end{array}$ \\
\hline
\end{tabular}

Réalisation de l'auteure.

\section{Des friches marquées par l'armée.}

Un premier grand ensemble comprend des bâtiments, en ruines ou non, qui conservent des traces de la présence militaire et notamment des pollutions spécifiques ou une architecture marquée. Ce sont des fortifications, des dépôts d'essence, des poudreries, des buttes de tir ou les terrains des bases aériennes.

Ces terrains, souvent à l'écart des voies de circulation, présentent également un intérêt architectural moindre pour une reconversion urbaine. On trouve parmi ces friches des éléments remarquables sur un plan patrimonial, tels certains forts de la ligne Maginot entretenus par des associations. De nombreux blockhaus restent également des verrues paysagères coûteuses à conserver car difficilement reconvertibles, quoique celui du village de Pouilly, sur la route de Metz, ait été récemment réhabilité en un atelier technique municipal de $525 \mathrm{~m}^{2}$ et que celui de Volgelsheim, construit en 1897 avec six salles voûtées soit proposé à la vente par la société Abedis, distributeur de boissons, qui en avait fait une vinothèque. D'autres sites ne peuvent être reconvertis du fait de leurs activités passées car ils conservent une forte valeur symbolique : le camp du Larzac a été dépollué et sécurisé mais ne peut être intégralement reconverti car l'armée veut conserver le secret des activités qui s'y sont déroulées.

De nombreux camps de tirs, terrains d'entraînement ou zones d'exercices complètent cet ensemble : éloignés des zones urbaines, ces sites sont fréquemment rendus à l'état de nature dès qu'ils sont dépollués et/ ou sécurisés, avec une prise en charge par l'Office National des Forêts (ONF). 


\section{Des biens assimilables à d'autres friches ?}

24 des logements ou des centres mobilisateurs. Ces sites, peu marqués par l'Armée et avec une certaine densité, datent souvent du XIXème siècle ; ils ont initialement été édifiés en lisière de ville, laquelle a progressivement disparu avec l'urbanisation et sont aujourd'hui en première couronne. La présence de ces implantations militaires crée de fortes discontinuités dans le tissu urbain, souvent des préfectures ou des souspréfectures : friches trouvent rapidement preneurs au prix du marché. Les infrastructures en plus mauvais état sont parfois cédées aux mairies ou à des établissements publics à des prix plus faibles. Ces immeubles représentent une bonne opportunité d'aménagement malgré la nécessité d'une importante réhabilitation.

Dans la majorité des cas, les actifs immobiliers du ministère des Armées concernent néanmoins de grandes casernes, souvent dans de petites communes. Ceci est fréquemment le principal obstacle à une cession rapide car l'absence d'un projet de reconversion à l'échelle de l'emprise et le défaut de financements sont problématiques. Ainsi, les quartiers Teyssier et Jouart (17 ha), composés de deux casernes occupées par l'armée jusqu'en 1996, localisés au nord-est de la zone agglomérée de Bitche et en limite de centre-ville, ont été complexes à reconvertir : les bâtiments cernant la place d'armes accueillent des services publics, une pépinière d'entreprises et du logement social tandis que les autres secteurs concernent de l'habitat individuel. Deux principes d'aménagement majeurs ont été validés en intégrant les besoins en matière de logements et d'équipements et l'état des bâtiments à conserver et ayant une valeur historique. Dans des villes plus importantes, ces immenses constructions restent des handicaps, en particulier lorsqu'elles sont classées et il arrive parfois que certains sites cumulent les inconvénients : Sedan, deuxième ville du département des Ardennes avec plus de 21000 habitants, est aussi l'une des plus pauvres de France avec un taux de chômage d'environ $26 \%$ dû à la forte désindustrialisation régionale. Son château, datant de 1424, est l'un des deux plus grands châteaux féodaux d'Europe; l'Armée l'utilisa jusqu'en 1962. Les lieux furent alors cédés à la ville pour un franc symbolique. Le secteur sauvegardé de Sedan, datant de 1992, comprend les 20 hectares du château et un secteur sauvegardé de 30 hectares ; le projet hôtelier de 1987 n'ayant pas trouvé les accords nécessaires, le château est resté à l'abandon. L'apport du label «ville d'art et d'histoire", en 2000 , a permis un nouveau dynamisme avec, d'une part, le secteur hôtelier qui a enfin pu prendre à sa charge la restauration de trois bâtiments dans le cadre d'un partenariat public-privé et, d'autre part, la rénovation de l'ensemble des quartiers de la ville dans le cadre d'un rééquilibrage de l'offre touristique. Aujourd'hui, le développement touristique a pérennisé le site et a redonné du dynamisme économique à la région, la population sedanaise décline, mais dans une logique régionale plus générale (Cochet, 2010).

Cette catégorie intègre les espaces destinés à la " vie sociale " des garnisons tels les cercles-mess ou les hôpitaux militaires. Moyennement densifiés, proches du centre urbain, dans des quartiers généralement destinés à l'habitat, aux commerces ou aux services dans les villes moyennes, ils se reconvertissent aisément dans la logique d'une friche tertiaire. Cependant, l'intérêt de ces bâtiments est parfois problématique :

Revue Géographique de l'Est, vol. 57 / 3-4 | 2017 
l'ancien hôpital militaire Gama, en périphérie du ban communal de Toul au sein d'un quartier résidentiel a été désaffecté en 1996. La Communauté de Communes du Toulois a ensuite sollicité l'EPF Lorraine pour différents travaux en vue d'une requalification. À partir de 2004, le site a pu être racheté par un promoteur privé pour une opération de 240 logements.

Parfois, le départ de l'armée est un bienfait pour la ville car il lui rend en quelque sorte l'intégralité de son urbanité. Certaines villes en ont profité bien avant que la structuration de la cession des friches militaires. Ainsi, Strasbourg a connu une histoire partagée entre la France et l'Allemagne, avec une forte implantation militaire qui cessa progressivement entre 1922 et 1958 , libérant près de 200 ha permettant à la ville différents aménagements : une salle des fêtes, le quartier Bourse, 75 hectares pour des habitations, des commerces et des bureaux. 17 hectares pour l'université et 13 hectares dédiés à l'aménagement du parc de la citadelle et à des logements, du moyen au haut standing pour valoriser la ville qui accueillait depuis peu les institutions européennes. L'hôtel du Département, achevé en 1989 complète cet ensemble et des ouvrages de fortification, pour partie réhabilités, accueillent notamment des ateliers d'artistes.

\section{Les emprises partiellement bâties à l'orée de la ville.}

Ce dernier ensemble comprend de grandes casernes, aérodromes ou centres de transmission avec de réelles perspectives d'aménagement du fait de leur localisation en zone urbaine constructible. Ils sont assimilables à des friches quelconques à l'exception de contraintes de pollution spécifiques. Ainsi, la communauté de communes de Verdun souhaitait requalifier le site militaire désaffecté du quartier Driant pour en faire une zone dédiée aux services et activités. Cette emprise, essentiellement constituée de hangars pour la maintenance des unités de chars a fait l'objet d'un accompagnement technique et financier de l'EPF Lorraine en 2001 puis la communauté de communes a reconverti le site en une zone d'activités artisanales et un pôle tertiaire.

En périphérie des villes, on trouve également des grands terrains d'exercice, non viabilisés et souvent à l'état de nature. Ils peuvent être utilisés pour de nouvelles constructions ou des aménagements paysagers qui participent à la qualité de vie urbaine. Ainsi, les anciens magasins militaires de Concentration (cinq bâtiments en mauvais état sur 1,5 ha au nord d'Epinal) ont permis d'améliorer la circulation automobile (destruction de deux bâtiments en 2007) et d'accueillir des associations dans les trois bâtiments restant en 2008.

\section{B. La cession des actifs immobiliers des Armées : une pratique originale?}

31 L'État met en vente un nombre croissant de biens immobiliers considérés comme des friches à partir de la cessation de leur activité initiale. Cette cession, liée à des démarches préalables, fait intervenir de nombreux acteurs une fois que le service utilisateur en déclaré l'inutilité. En l'absence d'exercice du droit de priorité d'achat par la commune sur le territoire de laquelle il est situé, la cession du bien concerné peut être réalisée selon le principe de la mise en concurrence. Les friches militaires mises en vente obéissent à ces principes généraux avec des adaptations. Cependant, alors que les ventes d'actifs immobiliers de l'État doivent contribuer au désendettement de l'État, le ministère des Armées a un régime dérogatoire qui lui permet de récupérer l'ensemble 
des produits de cessions jusqu'au 31 décembre 2019 afin de financer ses opérations extérieures.

Le premier enjeu pour le ministère des Armées est alors un pari opérationnel visant à rassurer et à accompagner les acheteurs potentiels. Il s'agit d'occulter la qualification de «militaire » de la friche en insistant sur ses atouts urbains et en démystifiant les activités qui y ont eu lieu. Il a donc été nécessaire d'adapter un outil immobilier suffisamment diversifié et intégrant les contraintes du secteur privé. À l'instar d'autres entreprises publiques elles-mêmes grands propriétaires fonciers, le ministère des Armées a su trouver une gestion immobilière appropriée. De fait, les textes réglementaires laissent aux Armées la possibilité d'initier les procédures d'aliénation. Une structure a été créée dans ce but en 1987, la Mission pour la Réalisation des Actifs Immobiliers (MRAI), qui mène, en concertation avec France domaine, les procédures de négociation des emprises en vente avec une intervention dans trois domaines :

- Favoriser des études de reconversion et d'urbanisme pour les sites les plus complexes,

- Mener des campagnes d'information sur des biens mis en vente auprès d'une sélection d'administrations, d'entreprises et de particuliers,

- Négocier la cession des biens pour concilier au mieux les intérêts des autorités locales et ceux du ministère de la défense.

Lorsque la cessation d'activité d'une emprise de la Défense est annoncée brutalement, les collectivités locales n'ont pas eu le temps d'intégrer la future friche dans un projet global pour la ville. La MRAI prend alors le relais en engageant un processus dont la finalité est d'éviter la vente à l'euro symbolique. Pour faire émerger un projet de reconversion durant le temps de l'avant-friche, le dossier est souvent confié à un urbaniste qui travaille de concert avec un comité de pilotage situé ad hoc et constitué du préfet, de représentants de la commune, de la région et du département, des principaux acteurs intéressés et des administrations.

Indépendamment des contraintes architecturales, la situation des friches militaires leur confère souvent une valeur territoriale. Les cités se sont construites autour d'elles, selon les usages et les représentations en vigueur et la caserne est fréquemment la première " entreprise » locale et, en dépit d'un fonctionnement autonome à l'écart de la communauté urbaine, une symbiose humaine et économique existe. De plus, la reconversion des terrains militaires permet un travail parfois exceptionnel entre les administrations et avec le secteur privé : l'urgence pour trouver une solution crée des intérêts communs avec la recherche de projets consensuels et l'adaptation des méthodes d'interface. La vocation publique des emprises succède souvent à son usage militaire avec une continuité de l'usage collectif qui donne en quelque sorte une image institutionnelle au nouvel occupant.

En complément des obligations génériques, pour la cession de ses sites, le ministère de la défense procède lui-même à la cessation d'activité et au démantèlement des installations classées qu'il a exploitées (Installations Classées pour la Protection de l'Environnement (ICPE) ou Installations, Ouvrages, Travaux et Activités classés (IOTA)), sauf si le nouvel acquéreur souhaite en conserver l'usage. La dépollution pyrotechnique reste un frein notable dans le processus de cession car l'état de pollution pyrotechnique présumé, puis, le cas échéant, avéré des terrains, crée des obligations préalables à la cession pour le ministère. Celles-ci ont récemment évolué. Ainsi, jusqu'en 2014, le décret n ${ }^{\circ} 76-225$ du 4 mars 1976 (attributions du ministre de la défense "en matière de recherche, de neutralisation, d'enlèvement et de destruction des munitions et des 
explosifs) avait été modifié pour permettre à l'autorité militaire cédant de transférer la réalisation de la dépollution pyrotechnique à l'acquéreur.

Cependant le ministre doit toujours faire examiner, avec une recherche historique, la situation des immeubles militaires pour les opérations de recherche, neutralisation, enlèvement et destruction des munitions, mines, pièges, engins et explosifs. Cette recherche historique (4 à 6 mois) répertorie et analyse les activités et les événements susceptibles d'avoir occasionné une pollution pyrotechnique du site. Le ministère de la défense a ensuite l'obligation de dépolluer, « en fonction de l'usage auquel ces terrains sont destinés » (article 4 du décret du 4 mars 1976 modifié), les emprises avant toute aliénation ou changement d'utilisation. Il est donc nécessaire, dès le temps de l'avantfriche, que le projet de reconversion des emprises cessibles soit suffisamment avancé. Pour l'acquéreur, les délais de réalisation des travaux constituent des éléments centraux dans la définition du projet de requalification du site. Néanmoins, pour accélérer les cessions d'emprises, l'acquéreur peut faire procéder lui-même à la dépollution, le coût de la dépollution s'imputant alors sur le prix de vente. L'État conserve la surveillance de ces opérations puisque la vente est subordonnée à leur réalisation.

Cependant, depuis 2014, le décret $n^{\circ} 76-225$ du 4 mars 1976 a été modifié pour mieux circonscrire le périmètre des engagements financiers de l'État vis-à-vis des acquéreurs privés. Ceci fait suite au cas de l'emprise militaire de la base aérienne 112 de Reims récemment mise sur le marché foncier. Pour l'agglomération de Reims, la fermeture du site, à l'été 2012, a pour conséquences le départ de plus de 3000 habitants et une perte de 500 emplois liée au manque à gagner généré par la consommation de cette population. Les 540 hectares de la base pouvaient être cédés à l'euro symbolique, mais la résolution du problème de pollution (risque pyrotechnique avéré lié à la présence dans le sol de munitions de la guerre de 1914-18, d'hydrocarbures de la guerre 1939-45 et d'amiante récente) atteignait les $30 \mathrm{M} €$. La pollution du terrain reste donc un frein à la reconversion de ce site dont 350 hectares sont devenus une ferme expérimentale en 2017. De fait, la disposition spécifique la plus conséquente du décret concerne le coût définitif de la dépollution pyrotechnique pris en charge par l'État qui ne pourra excéder le prix de vente du terrain, sauf dans le cadre des cessions faites au titre du dispositif dit de «l'euro symbolique».

\section{Le cadre financier de la reconversion des emprises militaires}

Les premières reconversions de sites militaires, avant les LPM, étaient trop marginales pour qu'un programme d'ampleur soit mis en place, bien que des aides génériques aient pu être apportées au niveau national. Pour les reconversions les plus récentes, l'État a mis en place un dispositif permettant aux communes les plus touchées de bénéficier à la fois d'études et de financements. Ceci s'inscrit dans une logique de compensation du départ des militaires, tous les sites concernés ne peuvent donc en bénéficier à l'identique. Ces aides se divisent en deux catégories : d'une part, des fonds généraux non dédiés aux sites militaires tels des Aides à Finalité Régionale (AFR), des exonérations fiscales et sociales ou des programmes régionaux; d'autre part, une organisation dédiée aux restructurations de la défense a été mise en place face à 
l'ampleur du nombre de sites militaires à reconvertir. Si certains organismes existaient avant les LPM, leur rôle a été reprécisé :

- Le Commissariat Général à l'Egalité du Territoire coordonne l'action d'accompagnement territorial avec celles des ministères concernés et instruit les contrats de sites ;

- La Délégations Aux Restructurations (DAR), secrétariat permanent du comité des restructurations du ministère des Armées, anime un réseau de 11 délégués territoriaux inter-régionaux, placés auprès des préfets de région. Ils sont les interlocuteurs des acteurs locaux;

- Le délégué ministériel à la mise en œuvre territoriale de la réforme anime et coordonne l'action du ministère de la défense dans les domaines de l'accompagnement des réorganisations au profit des personnels civils et militaires, mais aussi des restructurations au profit des territoires et du secteur économique ;

En parallèle, un dispositif d'aides et de soutien à la reconversion de sites militaires prévoit la réalisation obligatoire d'un diagnostic de territoire pour déterminer les actions à soutenir en priorité dans un objectif de redynamisation économique avec des mesures en faveur des entreprises et du soutien à l'emploi, en cohérence avec les autres politiques territoriales mises en œuvre par ailleurs par l'État. Les aides directes dans le cadre du dispositif national sont alors de deux types:

- Des Contrats de Redynamisation des Sites de Défense (CRSD) destinés aux sites subissant une perte nette de plus de 200 emplois directs et qui connaissent une grande fragilité économique et démographique ;

- Des Plans Locaux de Redynamisation (PLR) destinés aux sites dont la décision de transfert ou de dissolution entraîne une perte de plus de 50 emplois directs et qui subissent un impact significatif, mais réversible, sur l'économie locale.

Ces contrats sont signés avec les autorités locales pour une durée de trois ans, prolongeable de deux ans.

Sur la période 2009-2014, ces aides ont été conséquentes (300M€), tant en dotations et subventions aux collectivités locales que pour les entreprises s'implantant sur les territoires concernés et ont bénéficié à plus d'une cinquantaine de sites en France (Cour des Comptes, 2014). Enfin, un nouveau dispositif de prêts participatifs octroyés par la Banque Publique d'Investissement (BPI) au bénéfice des PME déjà situées dans les territoires touchés par les restructurations, ou ayant le projet de s'y implanter, a été instauré. L'étude « Les aides de l'État aux territoires concernés par les restructurations des armées » (ibid.) a analysé un échantillon de communes concernées par des contrats des sites (CRSD et PLR) dans le cadre de la LPM 2009-2014; le financement des projets est ainsi réparti :

- Les communes et intercommunalités financent seules $44 \%$ du total des projets inscrits ;

- L'État finance $26 \%$;

- Les 30\% restants proviennent de nombreux autres partenaires comme les régions, les départements, l'Union européenne, des organismes professionnels, des entreprises, etc.

\section{Quelles reconversions pour les friches militaires : le cas de la ville de Mulhouse}

Passé le premier traumatisme lié au départ des militaires et de leurs familles, les friches militaires urbaines revêtent parfois des dimensions si différentes qu'il est difficile 
d'envisager une généralisation quant à leur intégration dans les projets urbains. Néanmoins, à partir des reconversions qui ont été réalisées sur une centaine de sites, une dynamique de projet peut être retenue, en corrélation avec le besoin de renforcement d'attractivité et de dynamisme. Il s'agit d'exploiter une ressource foncière inopinée pour répondre aux besoins de la commune. Cette dynamique va spécifiquement intégrer les points positifs qui différencient la friche militaire urbaine d'autres ressources foncières disponibles sur la ville (doc. 3) : une localisation bénéficiant de réseaux de transports, des coûts de dépollution limités, des locaux réutilisables et enfin une aide technique et financière.

Document $\mathrm{n}^{\circ} 3$ : intégration de la friche militaire dans le projet urbain en rapport avec son urbanité.

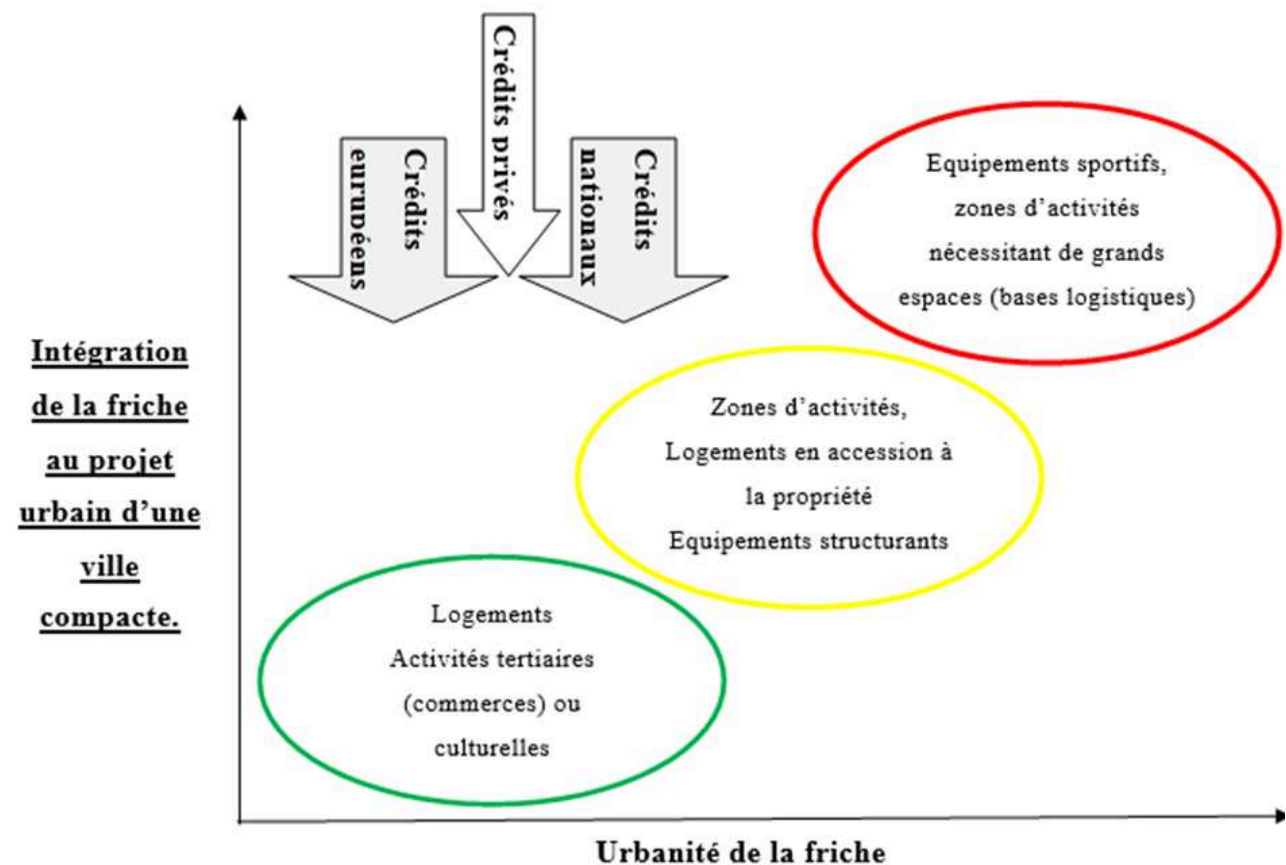

Distance de la friche par rapport au cœur de l'agglomération

Réalisation de l'auteur

Afin d'illustrer ces difficultés de reconversion, et notamment l'effet de surprise de planification urbaine, la ville de Mulhouse a été choisie pour présenter une typologie partielle de réussite de reconversion de site militaire.

Le développement urbain de Mulhouse reste marqué par la 1ère révolution industrielle, au milieu du XIXème siècle: la ville a connu une explosion démographique, sa population passant de 6000 à 100000 habitants en un siècle. De longue date, Mulhouse a été connue son cadre urbain de médiocre qualité dû à une croissance rapide et désordonnée et matérialisé par un tissu urbain avec une faible lisibilité : faute de terrains constructibles, l'extension s'est réalisée par des fusions communales imposées par une politique urbaine destinée à faciliter les grands aménagements urbains au rythme de la création des usines et de l'attraction de leur main-d'œuvre (Chassignet, 1994). Il en résulte une imbrication des bâtiments industriels et des quartiers d'habitations. Annexée par les Allemands en 1871, Mulhouse fut également une ville de garnison qui compta jusqu'à 5000 militaires pour 95000 habitants. Cinq casernes furent construites entre 1874 et 1909. Cependant, la présence des militaires a eu 
relativement peu d'impact sur l'urbanisme mulhousien du fait de la prédominance de l'industrie. Dans ce contexte de pression urbaine favorisant l'industrie, les premières reconversions de sites militaires furent des occasions de bénéficier de foncier pour des fonctions non strictement dédiées à l'industrie. Ainsi, la caserne Coehorn fut reconvertie en cité administrative à partir de 1946 puis devint la caserne des pompiers et le centre des impôts en 1973 et la caserne Brochet a été démolie en 1958 pour être incluse dans le site de construction du lycée Albert Schweitzer.

Mulhouse est aussi un cas emblématique de villes industrielles champignons (Duby, 1983) qui se désindustrialisent à partir de 1968 tout en gardant une forme de dépendance industrielle. Alors que la ville devait sa forte attractivité à la densité de son marché de l'emploi, les premiers chocs pétroliers eurent un effet immédiat. Les difficultés sociales se multiplièrent et s'accompagnèrent d'un profond malaise social avec des conséquences morphologiques telle la multiplication de friches industrielles, accentuant la désarticulation du tissu urbain, alors que la ville n'avait que peu de ressources financières pour les reconvertir (Chassignet, 1994).

Mulhouse a cependant entretenu une tradition de recyclage du foncier héritée de ses possibilités limitées de périurbanisation. Alors que la notion d'urbanisme durable s'imposait, la ville a donc utilisé ses friches et, dans les années 1990, elle initia une série de projets structurants pour redynamiser son centre-ville et développer de nouvelles polarités en reconvertissant notamment d'anciens sites industriels. De fait, la disparition quasi simultanée du rôle de ville de garnison et la libération par l'Armée d'une superficie totale de plus de 20 hectares en ville se sont alors réalisées dans l'indifférence tandis que, sur un plan socio-économique, la fermeture des trois derniers sites militaires avait un impact limité :

- La caserne Lefebvre abritait surtout du matériel et ne comptait que 20-25 personnes ;

- La caserne Barbanègre ferma en 1992 après une période de transition entre 1973 à 1987.

- La caserne Drouot, qui accueillait un petit détachement de militaires fut libérée en 1992.

Sur un plan urbain, la fin d'activité de ces casernes eut pour conséquences directes la fermeture puis la cession des lieux qui leur étaient liés, tels le cercle mess des officiers et la résidence du gouverneur militaire de Mulhouse, les logements réservés aux militaires ont quant à eux été rachetés par les bailleurs locaux. En 1993, à l'exception de ses bureaux de recrutement et de la Gendarmerie, Mulhouse ne comptait plus aucun militaire. Le besoin de compenser le départ des militaires n'était donc pas une priorité économique pour Mulhouse : la reconversion d'un site devait davantage s'inscrire dans une logique d'opportunité pour la ville, qui banaliserait la friche militaire pour la considérer comme une ressource foncière. Ceci est d'autant plus avéré que la municipalité était déjà engagée financièrement dans de grands projets tels la clinique et la faculté des sciences à la Fonderie ou l'équipement culturel du Nouveau Bassin non loin des casernes.

À partir de 2002, le Grand Projet de Ville a initié la rénovation des Quartiers anciens. La collectivité, par le biais de la Société d'Équipement de la Région Mulhousienne (SERM), tout juste créée, a utilisé les instruments d'urbanisme opérationnel tels les Zones d'Aménagement Concerté (ZAC) pour mener à bien ses projets. À cette période, Mulhouse connaît conjointement, sur du long terme, un processus d'affaiblissement économique, social et démographique et semble s'inscrire dans une logique de décroissance urbaine (Cunningham-Sabot et Fol, 2010). Celle-ci semble alors davantage marquée dans les anciens quartiers ouvriers avec des logements dégradés et le 
développement de friches urbaines. Ces quartiers deviennent des points de fixation de la crise (Miot, 2013).

49 La notion de "ville à deux vitesses » (Béhar, 1995) est de ce fait particulièrement présente dans les documents d'urbanisme rédigés au début des années 2000 pour lutter contre la périurbanisation et le départ des classes moyennes : il apparaît nécessaire de diversifier l'habitat et de valoriser le patrimoine. Alors que l'attrait initial pour la reconversion des trois dernières casernes mulhousiennes semblait limité, des changements de contexte ont pu renouveler leur intérêt pour la ville et son agglomération (doc. 4) :

Doc 4 : les casernes de Mulhouse, une situation favorable

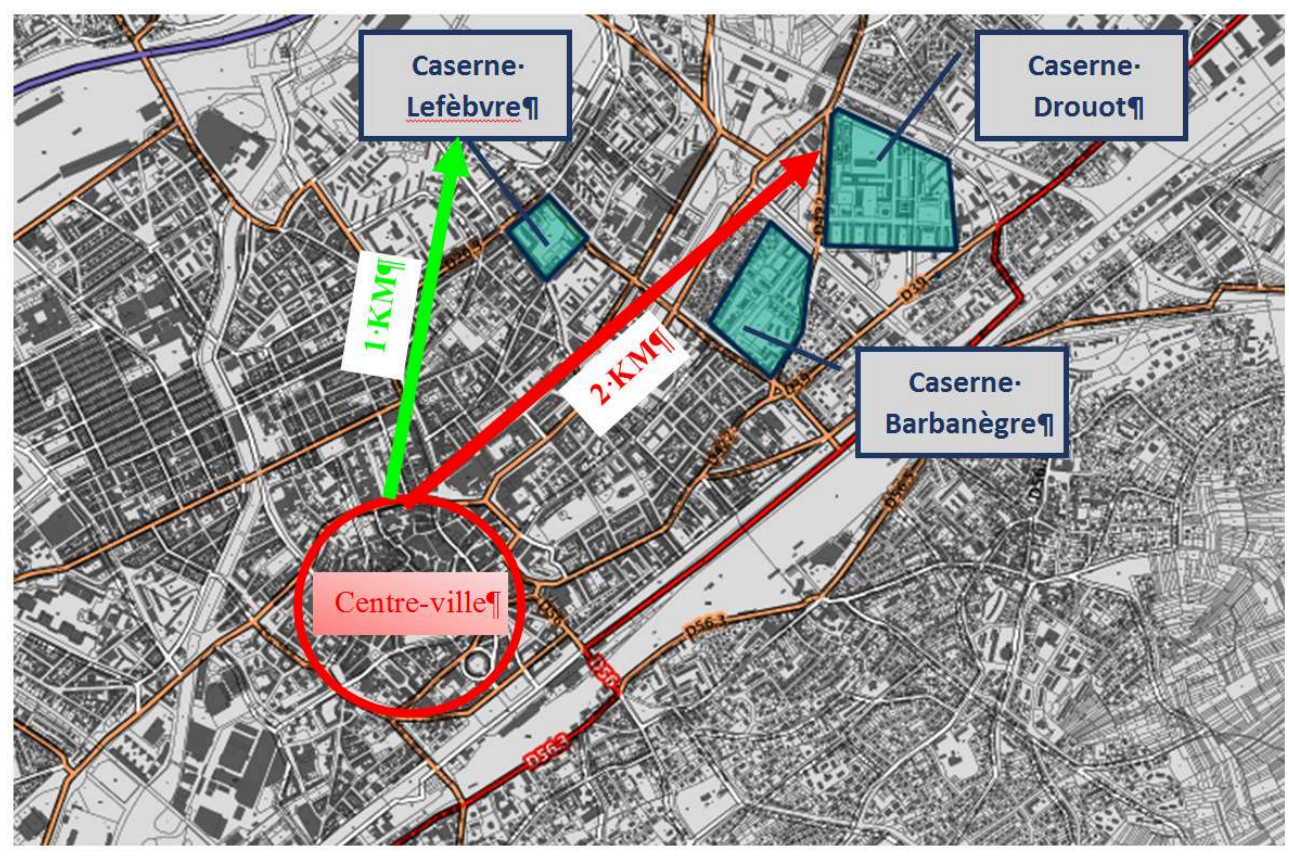

Source : Géoportail, habillage par l'auteure.

- La construction d'un réseau de tramway et du tram-train, décidée à la fin des années 1990, définit de nouvelles priorités dans l'aménagement urbain: le tracé des lignes pouvait valoriser les sites militaires.

- L'arrivée des TGV Est, puis Rhin-Rhône, incite la ville à engager un nouveau projet phare avec l'aménagement d'un quartier d'affaires autour de la gare et initie une réflexion sur l'éventuelle attractivité des autres sites en cours de de rénovation pour les inscrire dans une cohérence urbaine d'ensemble.

- Le développement de la politique de renouvellement urbain, avec l'appui de l'ANRU, dans les quartiers du péricentre, a généré un opportunisme pour les sites militaires situés dans le périmètre d'un quartier sensible éligible à des dispositifs de l'ANRU.

- À partir de 2000, la disponibilité de nouvelles friches industrielles, notamment le site de DMC) et la création d'une communauté d'agglomération d'environ 250000 habitants (INSEE, 1999) ouvrent de nouvelles perspectives de mixité fonctionnelle et de portage technicofinancier des projets. Ce changement d'échelle a pu donner une nouvelle impulsion à la place des casernes dans la dynamique de développement urbain de Mulhouse et de son agglomération.

Un processus de requalification a alors pu être mis en place pour chaque site. 


\section{La caserne Drouot}

51 Mulhouse et à $2 \mathrm{~km}$ du centre-ville, la caserne Drouot comprend 8,2 ha avec un environnement immédiat mixte : des logements sociaux en barre au sud et à l'Est, dont une partie des logements locatifs anciennement dédiés aux familles de militaires, des maisons individuelles accolées au nord et des équipements plus un semblant d'industrie à l'ouest. Les études menées par la SERM proposent un projet de requalification respectant une cohérence globale tout en conservant le bâti intéressant et en exploitant les surfaces disponibles. Acquis par la ville en 1997, le site a fait l'objet d'un programme mixte à dominante d'activité sous forme de ZAC clôturée le 31 décembre 2007. Il comprend des logements privés, des bureaux, des équipements publics et des locaux pour PME dans la partie village artisanal qui propose $8200 \mathrm{~m}^{2} \mathrm{de}$ surfaces dédiées aux petites entreprises et entreprises artisanales (doc. 5). Plus de 250 nouveaux emplois ont été créés et l'offre en services publics de proximité a été renouvelée. Il en est de même pour l'offre de logements. Ces résultats participent à l'effet levier de la reconversion de cette caserne : favoriser le retour d'une offre de services de proximité grâce à des locaux adaptés intra-muros et encourager le retour de catégories socio-professionnelles supérieures avec une offre variée de logements réhabilités.

Doc 5 : une allée du village artisanal dans les locaux réhabilités de la caserne Drouot

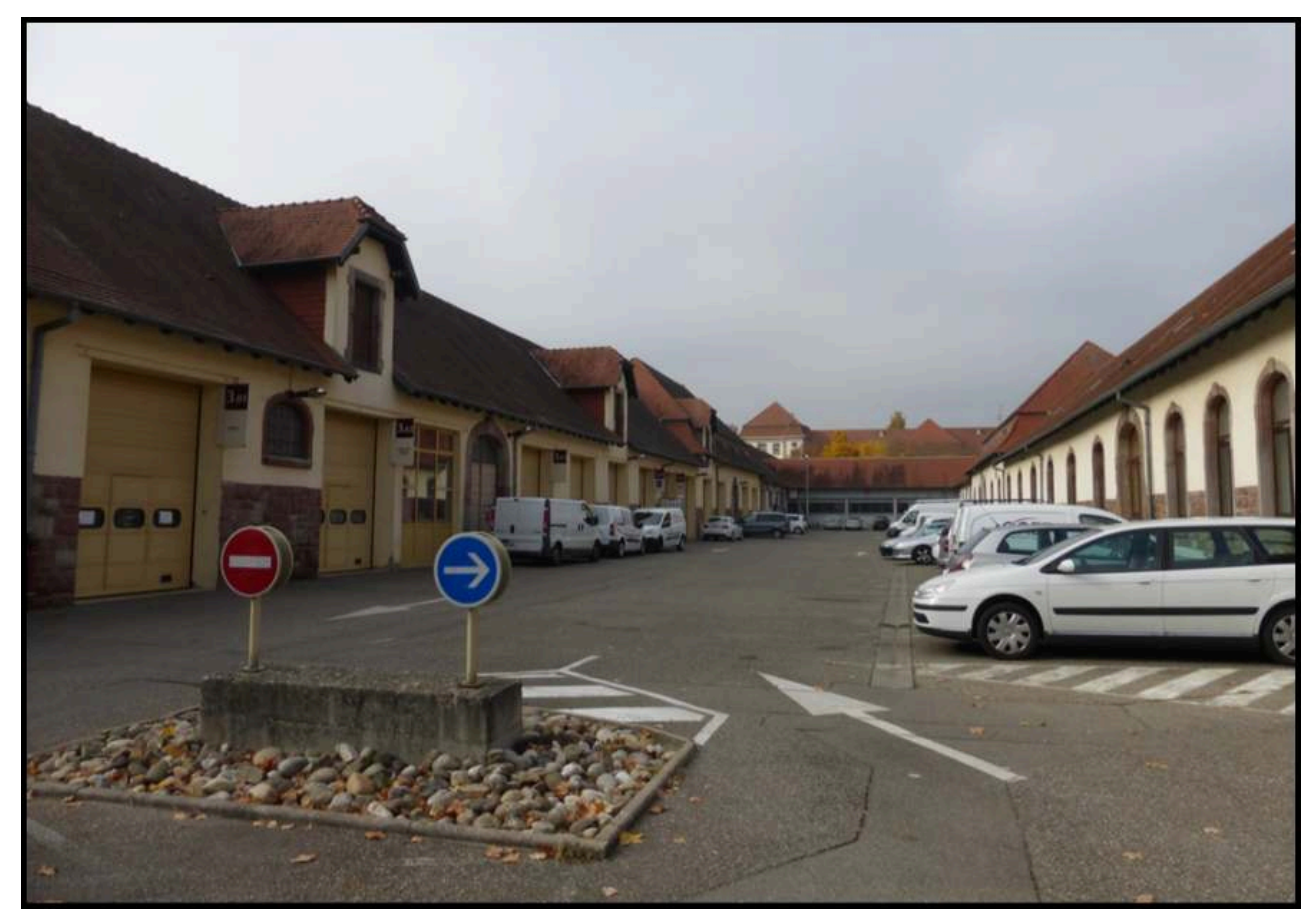

Cliché réalisé par l'auteur le 26 octobre 2015.

\section{La caserne Barbanègre :}

Construite à la même époque que la caserne Drouot, la caserne Barbanègre occupe un site plus vaste, 11 ha pour un bâti de $53400 \mathrm{~m} 2$, à $1.5 \mathrm{~km}$ de du centre-ville. Le site est 
divisé en deux parties: la caserne à proprement parler avec des bâtiments de logements et la zone dite technique avec des bureaux et d'imposantes structures initialement prévues pour l'accueil des chevaux. L'environnement même de la caserne est composé d'espaces verts. Le processus de reconversion a été identique à celui de la caserne Drouot avec une ZAC créée en juillet 2000 pour une dizaine d'années. À l'issue des travaux, le site reconverti, avec un grand espace vert, des bâtiments industriels, des locaux pour activités tertiaires, la gendarmerie départementale avec ses logements associés et deux îlots de logements, appartements ou maisons jumelées en accession à la propriété a renouvelé l'attractivité et le dynamisme du quartier : il offre un cadre sécurisant pour des catégories socio-professionnelles supérieures qui souhaitent habiter non loin du centre-ville (doc. 6). Cette offre complète l'effet levier de la caserne Drouot avec des services de proximité facilement accessibles.

Document $n^{\circ} 6$ : La caserne Barbanègre, une reconversion en logements de qualité.

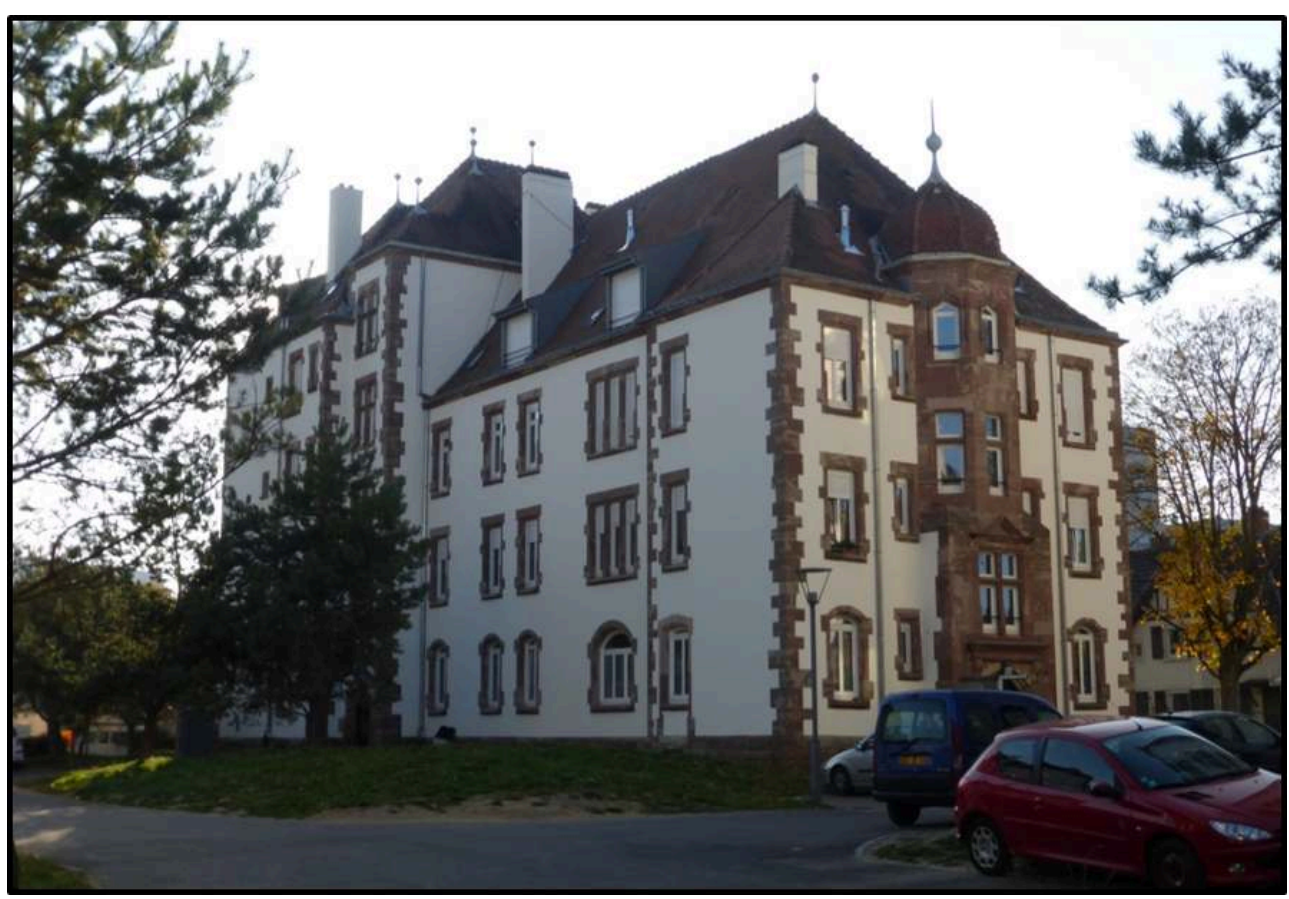

Cliché réalisé par l'auteur le 26 octobre 2015

\section{La caserne Lefebvre:} successivement incarné la nouvelle puissance prussienne qui l'érigea entre 1874 et 1877, l'héroïsme des soldats français dans les combats de la Libération en 1944 et enfin le passage obligé du service militaire après-guerre en comme Centre Mobilisateur jusqu’à sa désaffectation.

La caserne Lefebvre, située sur quatre hectares à $1,5 \mathrm{~km}$ du centre-ville, constitue un espace essentiel dans l'organisation du quartier. De nombreux projets de reconversion ont été étudiés sans concrétisation car la reconversion présentait ab initio de nombreux freins tels sa localisation géographique dans le périmètre de l'église Sainte-Jeanned'Arc, partiellement classée, et la structure même de la caserne dont le bâti semblait peu adapté à une réhabilitation (doc. 7). 
Document $\mathrm{n}^{\circ} 7$ : le site reconverti depuis l'ancienne place d'armes avec un aménagement paysager

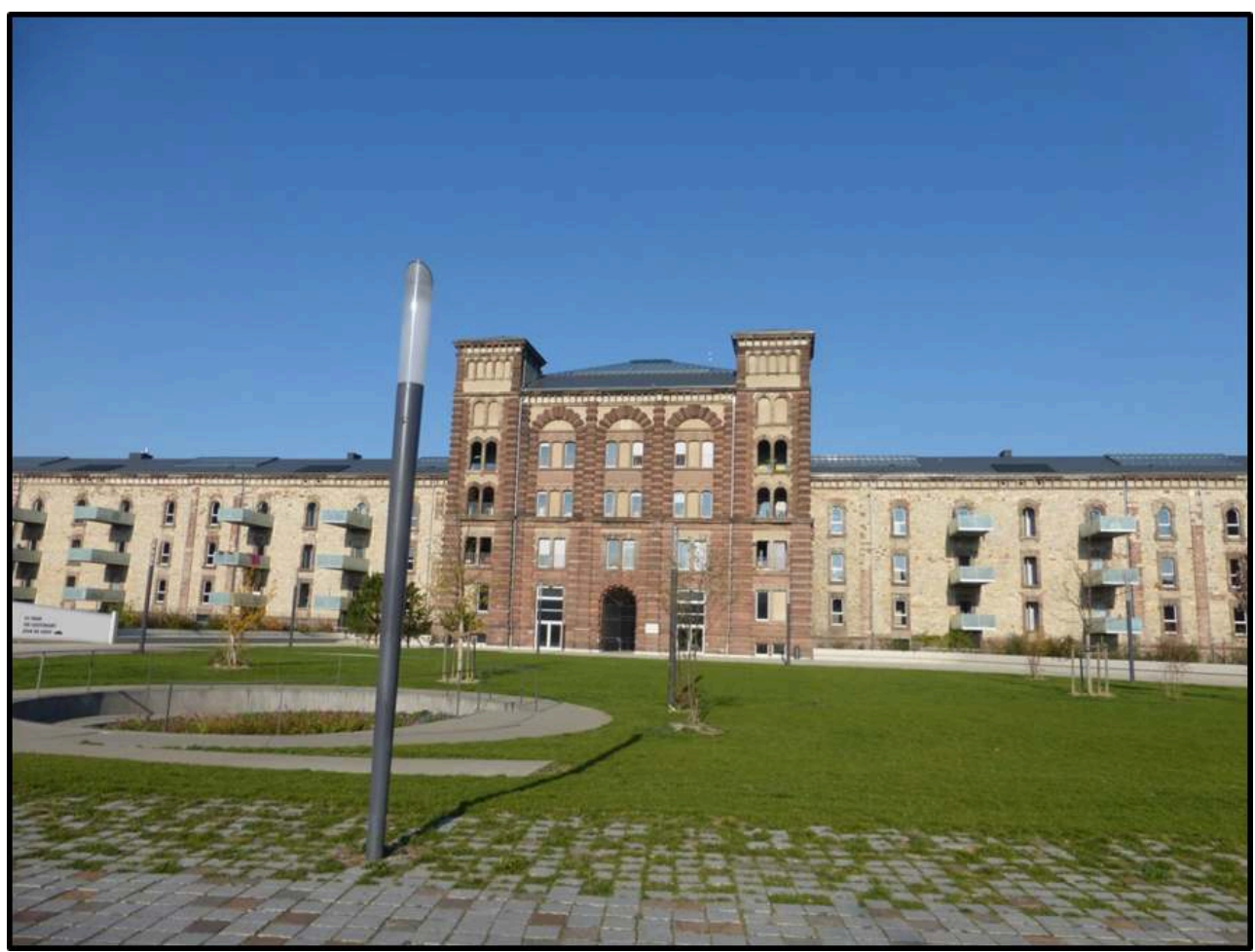

Cliché réalisé par l'auteur le 26 octobre 2016

L'opération d'aménagement finalement retenue s'est inscrite dans une démarche globale de renouvellement urbain au profit de l'ensemble du quartier pour répondre à plusieurs objectifs complétant l'effet levier de la reconversion des deux autres casernes :

- La mixité sociale par le biais d'une offre de logements neufs de qualité ;

- Une amélioration de l'image du quartier et du cadre de vie de ses habitants avec la production d'espaces publics de qualité ;

Sur le principe des deux autres opérations d'aménagement de casernes, la réhabilitation de la caserne Lefebvre a été réalisée dans le cadre d'une ZAC. Cette requalification (doc. 7) s'est effectuée dans le contexte d'une réflexion englobant tout le quartier Vauban/Neppert, pour une superficie totale de 25ha. Après dix ans d'attente, la caserne Lefebvre a été intégralement reconvertie en huit ans. Elle constitue le marquage urbain du projet de requalification du quartier dessiné par Nicolas Michelin.

Tout comme pour les casernes Drouot et Barbanègre, la reconversion du site Lefebvre a eu un effet levier au niveau du quartier, d'autant plus que celui-ci bénéficie d'une desserte par le tramway.

En faisant revenir en ville des habitants qui avaient choisi la périurbanisation, tout en renouvelant l'offre de services et la qualité de vie, la reconversion de ces trois casernes a permis à Mulhouse d'apporter un ensemble de réponses à la décroissance urbaine tout en s'inscrivant dans une logique de mixité fonctionnelle. Cependant, il est difficile d'attribuer un effet levier à la seule requalification des sites militaires car d'autres opérations d'urbanisme engagées peu d'années auparavant ou simultanément, donc toutes contemporaines de l'aménagement de ces friches militaires, peuvent avoir 
contribué à cet effet levier pour la ville. Ces friches militaires reconverties ont donc participé à l'atteinte des objectifs du projet urbain, ne se démarquant pas fonctionnellement des autres friches industrielles ou tertiaires qui y ont contribué.

\section{Conclusion}

Alors que les conséquences territoriales de la prochaine LPM devraient être prochainement officialisées, un focus sur les friches militaires a permis de banaliser celles qui sont situées en ville et qui peuvent représenter une opportunité foncière dans le cadre de la ville durable tandis que précisément le ministère des armées facilite la reconversion de ces sites. De fait, des éléments généraux, applicables à toutes les reconversions de friches, caractérisent les requalifications de sites militaires avec d'une part, la nécessité de définir un besoin que la reconversion de la friche pourrait satisfaire dans le cadre d'un projet urbain d'ensemble, avec, de facto, la nécessité d'appréhender le quatrième temps de la friche reconvertie, et, d'autre part, le besoin de bénéficier d'une structure adéquate pour porter la reconversion. L'intégration et la mise en pratique de ces deux éléments peuvent constituer des facteurs de réussite de la reconversion de sites militaires urbains.

L'exemple de la reconversion des sites militaires de Mulhouse a par ailleurs montré qu'au-delà de la requalification en elle-même, il s'agissait de s'interroger sur la capacité d'une ville, quelle que soit sa taille, à faire face à une surprise de planification urbaine et à intégrer sa requalification avec cohérence dans son projet urbain. Ainsi, la métropole alsacienne, engagée dans des opérations ou programmes qui mobilisaient ses ressources financières, a dû envisager des solutions d'attente qui ne remettaient pas en question le succès des autres opérations urbaines en cours de réalisation, tout en permettant aux édiles d'assumer leurs responsabilités face aux friches. Cette problématique est d'autant plus avérée pour des petites ou moyennes villes confrontées à des fermetures de sites importants.

\section{BIBLIOGRAPHIE}

Ambrosino C., Andres L., 2008, « Friches en ville: du temps de veille aux politiques de l'espace. », Espaces et sociétés, $\mathrm{n}^{\circ} 134$, p.37-51, consulté le 17 juin 2013. URL : www.cairn.info/revue-espaceset-societes-2008-3-page-37.html

Behar, D., 1995, « Banlieues ghettos, quartiers populaires ou ville éclatée ? - L'espace urbain à l'épreuve de la nouvelle question sociale », Annales de la recherche urbaine, p.68-69

Carré C, 2001, Histoire du Ministère de la Défense, Paris, éditions Lavauzelle, 582p.

Chassignet D., 1994, Mulhouse : centralité et polarités ", Revue Géographique de l'Est, $\mathrm{n}^{\circ} 2$, tome 34, 1994, Villes de l'Est français. p.101-116, consulté le 17 juillet 2016, URL : www.persee.fr/web/ revues/home/prescript/article/rgest_0035-3213_1994_num_34_2_2272 
Cochet L., 2010, « « Un partenariat public-privé pour sauver et valoriser le château de Sedan » in Corinne Berneman et Benoît Meyronin (dir.)Culture et attractivité des territoires, Nouveaux enjeux, nouvelles perspectives, Paris, L'Harmattan, p.221-232

Cohen-Grillet P., 2008, « Un plan pour les villes qui vont perdre leur garnison », France Soir, 17 septembre

Duby G., Agulhon M (dir.), 1983, Histoire de la France urbaine. T.4, la ville de l'âge industriel : le cycle haussmannien, Paris, Seuil, coll. L'Univers Historique, 494p.

Fol S., Cunningham-Sabot E., 2010, « Déclin urbain et Shrinking Cities : une évaluation critique de la décroissance urbaine ", Annales de Géographie, n674, p.359-383.

Grellety-Bosviel A., 1970, « Aménagement du territoire et politique immobilière des armées », Le moniteur des travaux publics et du bâtiment, $\mathrm{n}^{\circ} 41, \mathrm{p} .16-20$.

Guelton S., 1998, « Dix années de vente des terrains militaires », Études foncières, n 79, p.19-20.

Guelton S., 2001, « Les communes face à la reconversion des sites militaires », Études foncières, vol. 93,

Janin C., Andres L., 2008, « Les friches : espaces en marge ou marges de manœuvre pour l'aménagement des territoires? ", Annales de géographie 5/2008, nº663, p.62-81, consulté le 17 juin 2013, URL: www.cairn.info/revue-annales-de-geographie-2008-5-page-62.htm

Lacaze J-P., 2006, La transformation des villes et les politiques urbaines : 1945-2005, Paris : Presse de l'ENPC, 255p.

Lotz S., 2017, « L'effet levier de la reconversion des friches militaires dans le cadre du renouvellement urbain en France ", thèse de doctorat en aménagement de l'espace et en urbanisme sous la direction de Guelton S., Lab'urba, École d'Urbanisme de Paris, Paris 12, 2 tomes, 241 et $427 \mathrm{p}$.

Miot Y., 2013, « Renouveler l'habitat des quartiers anciens dans le cadre de la « Politique de la Ville » : la gentrification comme horizon ? - Les exemples de Mulhouse, Roubaix et Saint-Étienne ", Métropoles, 13|2013, mis en ligne le 10 janvier 2014, consulté le 8 juin 2016. URL : http:// metropoles.revues.org/4777.html

Piron Olivier, 2002, Renouvellement urbain, analyse systémique, Paris-La Défense : METL-PUCA, 133p.

Regrain R., 1988, « Les territoires de l'armée en France métropolitaine », collection 1988. Consulté le 25 février 2013, URL : www.mgm.fr/PUB/Mappemonde/M188/p38-41.pdf Robins des villes 2008, Les friches industrielles : cartographie et modes d'occupation, Lyon, Éd. du CERTU, 47p.

Sites internet consultés :

Adéquations.org, www.adequations.org, 3 juin 2018

Forum Vauban, http://www.vauban.de / http://www.forum-vauban.de, 7 février 2015

Sga.defense.gouv.fr, www.defense.gouv.fr/sga, 20 avril 2014

Sud-ouest.fr, www.sudouest.fr/, 5 août 2010

Vauban : http ://< www.quartier-vauban.de, 7 février 2015 


\section{NOTES}

1. Anciennement ministère de la Défense de 1974 à 2017.

2. https://www.freiburg.de/pb/site/Freiburg/get/params_E804213746/647920/ Infotafeln_Vauban_fr.pdf, consulté le è février 2015.

3. Adéquations.org, www.adequations.org, consulté le 3 juin 2018.

4. Sud-ouest.fr, www.sudouest.fr/ édition du 5 août 2010, consulté le 22 mars 2016.

5. Soit environ 38 millions d'euros.

\section{RÉSUMÉS}

Alors que la ville, autrefois gourmande d'espace foncier sur ses franges, s'efforce aujourd'hui de rationaliser sa trame urbaine dans une logique de développement durable, elle s'intéresse davantage à ses espaces délaissés. Parmi ces sites en attente de reconversion, les fiches militaires offrent de réelles opportunités de requalification et participent ainsi au renouvellement urbain. Cet article propose notamment d'envisager l'éventuelle spécificité de ces friches tout en complétant des notions déjà connus sur le foncier en attente de requalification. Le terrain d'étude de Mulhouse, avec trois casernes reconverties, sera ensuite analysé afin d'illustrer ces éléments.

In the past, the city used easily the land space on its fringes. While it is today trying to rationalize the urban network in the frame of sustainable development, it is more interested in its derelict lands and it tries to set up a frame of urban regeneration, which could be applied to all these wastelands. Among these waiting for renewal sites, the urban military brownfields could give real opportunities as part of urban regeneration and so participate to the urban renewal. This article proposes to consider the possible specificities of these brownfields while complementing already known notions about the waiting for requalification lands. The city of Mulhouse with three converted barracks will then be analyzed to illustrate these elements.

Die Stadt war früher raumsgierig auf ihren Fransen aber heute versucht sie in einer Logik der dauerhaften Entwicklung, den Stadteinschlag, zu straffen. Sie interessiert sich doch mehr für ihre verlassenen Räume. Unter diesen in der Requalifizierungerwartung Gegenden bieten die militärische Ödländer wirkliche Wiedernutzbarmachung für die Stadterneuerung an. Dieser Artikel schlägt insbesondere die Mögliche Spezifität dieser Ödländer vor, während bereits bekannte Konzepte auf dem Land zur Requalifikation abgeschlossen werden. Das Studiengelände von Mulhouse mit drei umgebauten Baracken wird dann analysiert, um diese Elemente zu veranschaulichen. 


\section{INDEX}

Schlüsselwörter : militärische Ödland, Stadterneuerung, städtisches Projekt, Hebelwirkung, Requalifizierung von Ödland

Mots-clés : friches militaires, renouvellement urbain, projet urbain, effet levier, reconversion de friches

Keywords : military brownfields, urban renewal, urban project, leverage effect, brownfield regeneration.

\section{AUTEUR}

\section{STÉPHANIE LOTZ-COLL}

Docteure en aménagement de l'espace et en urbanisme

Chercheur associé au Lab' urba, école d'urbanisme de paris Université Paris-Est (Paris 12)

stephanie.lotz.urba@gmail.com 